\title{
Management of diabetic ketoacidosis following implementation of the JBDS guidelines: Where are we and where should we go?
}

\author{
WINSTON CRASTO, ${ }^{1}$ ZIN ZIN HTIKE, ${ }^{2}$ LISA TURNER, ${ }^{3}$ KATH HIGGINS4
}

\begin{abstract}
Background: The Joint British Diabetes Society (JBDS) consensus guideline published in $\mathbf{2 0 1 0}$ has provided the framework for the effective management of diabetic ketoacidosis (DKA) in adults in the UK.

Methodology: A retrospective study of 50 patient episodes admitted to our teaching hospital between February and December 2012, with a discharge diagnosis of DKA.

Results: Twenty-seven (54\%) patients were male, mean (SD) age was 43 (21) years and duration of diabetes was 11 (9) years. In the first 60 minutes from diagnosis, median (interquartile range [IQR]) time to fixed rate intravenous insulin infusion (FRIII) was 49 (29-110) minutes and to intravenous fluids was 19 (0-42) minutes. During ongoing management, $46 \%$ of patients developed hypokalaemia and, of those, in $70 \%$ potassium supplementation was not prescribed as per protocol. Forty percent of patients experienced hypoglycaemia in the first 24 hours, of whom $80 \%$ had $10 \%$ dextrose prescribed appropriately according to protocol. Median time to hypoglycaemia from diagnosis was 12 hours 54 minutes. Median (SD) time to resolution of DKA was 12 hours 6 minutes. Eighty six percent of patients were reviewed by the diabetes specialist team during admission. No deaths due to DKA or complications of its management were reported. Median length of hospital stay was two days.
\end{abstract}

Conclusions: Adherence to the JBDS DKA guideline was

Registrar in Diabetes \& Endocrinology, University Hospitals of Leicester NHS Trust, Leicester, UK

Speciality Registrar and Clinical research fellow, Leicester Diabetes Centre, University Hospitals of Leicester NHS Trust, Leicester, UK

Consultant Diabetes \& Endocrinology, Kettering General Hospital NHS Foundation Trust, Kettering, UK

${ }^{4}$ Consultant in Diabetes, University Hospitals of Leicester NHS Trust, Leicester, UK

Address for correspondence: Dr Kath Higgins

University Hospitals of Leicester NHS Trust, Infirmary Square, Leicester, LE1 5WW, UK.

E-mail: kath.higgins@uhl-tr.nhs.uk

http://dx.doi.org/10.15277/bjdvd.2014.040

\author{
Abbreviations and acronyms \\ $A B C D \quad$ Association of British Clinical Diabetologists \\ CBG capillary blood glucose \\ DKA diabetic ketoacidosis \\ FRIII fixed rate intravenous insulin infusion \\ ICU intensive care unit \\ JBDS Joint British Diabetes Society \\ NaDIA National Diabetes Inpatient Audit \\ NICE National Institute for Health and Care Excellence \\ UHL University Hospital of Leicester
}

good in the immediate stage of treatment. Inadequate metabolic monitoring, fluid management and iatrogenic hypoglycaemia remain areas of concern. A high proportion of patients received diabetes specialist nurse input with reduced length of stay and no recorded mortality. Our recommendations as a result of this audit include a strengthened programme of teaching and education for nursing and medical staff, focus on metabolic monitoring and improved patient contact after hospital discharge.

Br J Diabetes Vasc Dis 2015;15:11-16

Key words: diabetic ketoacidosis, Joint British Diabetes Society, diabetes mellitus, in-patient care

\section{Introduction}

DKA is an acute metabolic emergency occurring in individuals with absolute or relative insulin deficiency and is typically characterised by hyperglycaemia, ketonaemia and acidosis. Untreated or mismanaged, DKA can potentially lead to fatal complications and emerging data indicate that this is inextricably linked to the quality of hospital care. ${ }^{1}$

DKA is commonly associated with type 1 diabetes and poses a significant threat at the time of diagnosis. ${ }^{2,3}$ Amidst growing concerns about the rising burden of type 1 diabetes, incidence trends predict a doubling of newly diagnosed cases in Europe by 2020. ${ }^{4}$ However, the incidence of DKA at diagnosis of type 1 diabetes in the UK has remained unchanged at around 25\% over the past 20 years. ${ }^{5}$ Data from the Diabetes Paediatric Audit in 2010 showed that $15.5 \%$ of children and young people had experienced one episode of DKA in the last five years and $10.4 \%$ had two or more events in the last five years. ${ }^{6}$ Furthermore, nearly $31 \%$ of individuals with type 1 diabetes in the UK were 
readmitted with DKA within a year from their initial hospital admission. ${ }^{7}$ A recent US study showed that period prevalence estimates of DKA in individuals with type 1 diabetes over the last decade were high at around $30 \%$ but remained stable over time. ${ }^{8}$

In March 2010, the JBDS published guidelines (subsequently revised in September 2013) on the management of DKA in adults. These guidelines were rapidly introduced by diabetes hospital teams across the UK and accepted as the national guideline for managing DKA. ${ }^{7}$ It was anticipated that such a move should significantly improve the management of people admitted with DKA which, prior to 2010, was deemed to be inconsistent with wide variations of care between hospitals in the UK. ${ }^{7}$ Key features included use of a FRIII, continuation of background (long acting/intermediate acting) insulin, preference for venous blood sampling, bedside blood ketone monitoring and commencement of $10 \%$ dextrose solution when blood glucose levels fall to less than $14 \mathrm{mmol} / \mathrm{L}$ alongside crystalloid fluid replacement on FRIII treatment. Subsequent to these developments, the NaDIA 2012 suggested that nearly $78 \%$ of participating hospitals had either implemented or modified the JBDS DKA guidelines. 9,10

In February 2012, led by the Adult Inpatient Diabetes Steering Group, the UHL NHS Trust implemented a revised DKA guideline based on the JBDS DKA guidelines. ${ }^{11}$ Implementation of the guideline was supported by a dedicated DKA prescription and monitoring chart. It was agreed locally to retain the priming bolus dose of insulin since there was uncertainty about the timing of intravenous insulin administered to patients with DKA in the initial hours after admission to the UHL Emergency Department. A retrospective audit of the management of adult patients with DKA admitted to UHL using our own revised DKA protocol as the gold standard comparator of care, was conducted to review adherence and assess improvements in quality of care.

The aims of this audit were:

1. To assess adherence to DKA guidelines in patients managed according to a dedicated DKA prescription and monitoring chart available within a hospital setting.

2. To analyse outcome measures including immediate management up to 60 minutes after hospital admission, on-going management from 60 minutes to 24 hours, and treatment induced hypoglycaemia.

3. To assess the effectiveness of management including time to resolution of DKA, time to conversion to subcutaneous insulin, and length of hospital stay.

\section{Methods}

UHL NHS Trust is one of the six biggest hospital Trusts in the UK, treating in excess of 1 million patients per year. The Leicester Royal Infirmary, one of three hospital sites within UHL, provides the main Accident \& Emergency service. We retrospectively reviewed case notes of all adult patients ( $\geq 17$ years of age) admitted at the Leicester Royal Infirmary, who were identified using a coded discharge diagnosis of DKA between February 2012 and December 2012. All data obtained from patient med- ical records were anonymised and stored in password protected files in keeping with principles of information governance. Data were collected using an audit tool, modelled from the NICE inpatient foot audit tool and with standards derived from the JBDS DKA guidance. ${ }^{7}$ The audit tool covered all aspects of management from admission to discharge and adherence to the protocol/use of dedicated hospital DKA prescription and monitoring chart and clinical outcomes.

\section{Definitions}

DKA was defined as a blood glucose $>11 \mathrm{mmol} / \mathrm{L}$ or known diabetes mellitus, ketonuria ( $>2+$ on standard urine sticks) or ketonaemia $>3.0 \mathrm{mmol} / \mathrm{L}$, venous $\mathrm{pH}<7.3$ and/or bicarbonate $<15 \mathrm{mmol} / \mathrm{L}$. Hypoglycaemia was defined as a blood glucose $<4.0 \mathrm{mmol} / \mathrm{L}$. Resolution of DKA was defined as $\mathrm{pH}>7.3$, bicarbonate $>15.0 \mathrm{mmol} / \mathrm{L}$, and blood ketone level $<0.3 \mathrm{mmol} / \mathrm{L}$.

\section{Biochemical measures and statistics}

All biomedical measures were carried out using standard operating procedures in the biochemical laboratory, UHL. Data were recorded on a Microsoft Excel spreadsheet. Data analysis was carried out using SPSS version 20.0. We report continuous variables as mean, median, interquartile range and standard deviation (SD). Categorical variables are reported as counts and percentages. Univariate analysis was performed using t-test for continuous and chi-square test for categorical variables. Spearman's correlation test was used to measure the strength of association between variables; $\mathrm{p}<0.05$ was considered.

\section{Results}

\section{Characteristics of patients}

One hundred and seventy-two episodes were identified with a coded discharge diagnosis of DKA. Of these, we accessed 100 patient case records. Fifty episodes were excluded from the audit ( $n=17$, not using the hospital prescription and monitoring chart [proforma]; $n=5$, confirmed diagnosis but protocol not used; $\mathrm{n}=28$, incorrect coded diagnosis). Among the 17 patients in whom the proforma was not used, 11 were admitted directly to the ICU where the proforma was not in use, four received a variable insulin infusion and, in the remaining two, recording on the proforma was stopped after ICU admission. Incorrect diagnoses included patients admitted with lactic acidosis $(n=2)$, impending DKA $(n=11)$, hyperglycaemia $(n=5)$ and other diagnoses $(n=10)$. Therefore, 50 correctly coded DKA patient episodes (44 single patient episodes, three episodes relating to two patients each) in which the hospital proforma was followed were used in the audit. Each episode was audited as a discrete event. Baseline characteristics of patients are shown in Table 1. Twenty-seven (54\%) patients were male and 23 (46\%) were female. Mean (SD) age and duration of diabetes of patients was 43 (21) years and 11 (9) years, respectively.

Diagnosis and immediate management (0-60 minutes) Median time (interquartile range, IQR) to diagnosis from the 
Table 1 Baseline characteristics of patients included in the DKA audit

\begin{tabular}{|c|c|}
\hline Mean age, years (SD) & $43(21)$ \\
\hline Males, n (\%) & $27(54 \%)$ \\
\hline Females, n (\%) & $23(46 \%)$ \\
\hline Mean duration of diabetes, years (SD) & $11(9)$ \\
\hline \multicolumn{2}{|c|}{ Number of hospital admissions in last 12 months, $\mathrm{n}(\%)$} \\
\hline 0 & $25(50 \%)$ \\
\hline 1 & $13(26 \%)$ \\
\hline 2 & $2(4 \%)$ \\
\hline 3 & $4(8 \%)$ \\
\hline$\geq 4$ & $6(12 \%)$ \\
\hline Mean length of hospital stay, days (SD) & $3.2(3)$ \\
\hline
\end{tabular}

point of entry to the emergency department was 25 (13-55) minutes. After the diagnosis of DKA was confirmed, a stat dose of subcutaneous or intramuscular insulin was administered at a median (IQR) time of 29 (14-64) minutes, FRIII commenced at 49 (29-110) minutes and intravenous fluids were commenced at $19(0-42)$ minutes. Within $0-60$ minutes, $74 \%$ and $78 \%$ of patients received intravenous fluids and FRIII, respectively. Beyond the 0-60 minute period, median time for administration of both intravenous fluids and FRIII was 75 (67-125) minutes. Seventy-six percent of patients were managed in an appropriate clinical area (Level 1+ as a minimum) (Figure 1).

\section{Ongoing management (1-24 hours) \\ Potassium replacement}

In the first 24 hours, serum potassium levels were within the normal range in $27 / 50$ (54\%) patients (mean baseline potassium at admission $5.13 \mathrm{mmol} / \mathrm{L}$ ). Potassium replacement was undertaken according to protocol in $42 \%$ of patients with no evidence of hyperkalaemia in any patient. Forty-six percent of patients developed hypokalaemia and in nearly $70 \%$ of patients of this group, potassium supplementation was not prescribed as per protocol. The average lowest potassium level was $3.1 \mathrm{mmol} / \mathrm{L}$ and lowest documented serum potassium was $2.5 \mathrm{mmol} / \mathrm{L}$. Hypokalaemia was managed according to hospital guidelines and no evidence of harm (clinically untoward incident) was documented as a result. Thirty-four patients (68\%) had $\leq 3$ interval serum potassium checks and 16 patients $(32 \%)$ had $>3$ interval serum potassium checks in the period 1-24 hours after admission. Of the latter, only two patients had all of the seven interval checks (by venous blood gas) as recommended in the protocol. Hypoglycaemia

Forty percent of patients experienced hypoglycaemia during the 1-24 hour management stage. The median time to hypoglycaemia from diagnosis was 12 hours 54 minutes. The lowest mean $C B G$ reading was $3.0 \mathrm{mmol} / \mathrm{L}$ and the lowest documented CBG was $2.0 \mathrm{mmol} / \mathrm{L}$. Eighty percent of patients had appropriate
Figure 1. Initial care processes occurring (0-60 minutes) after diagnosis in the management of DKA as per protocol

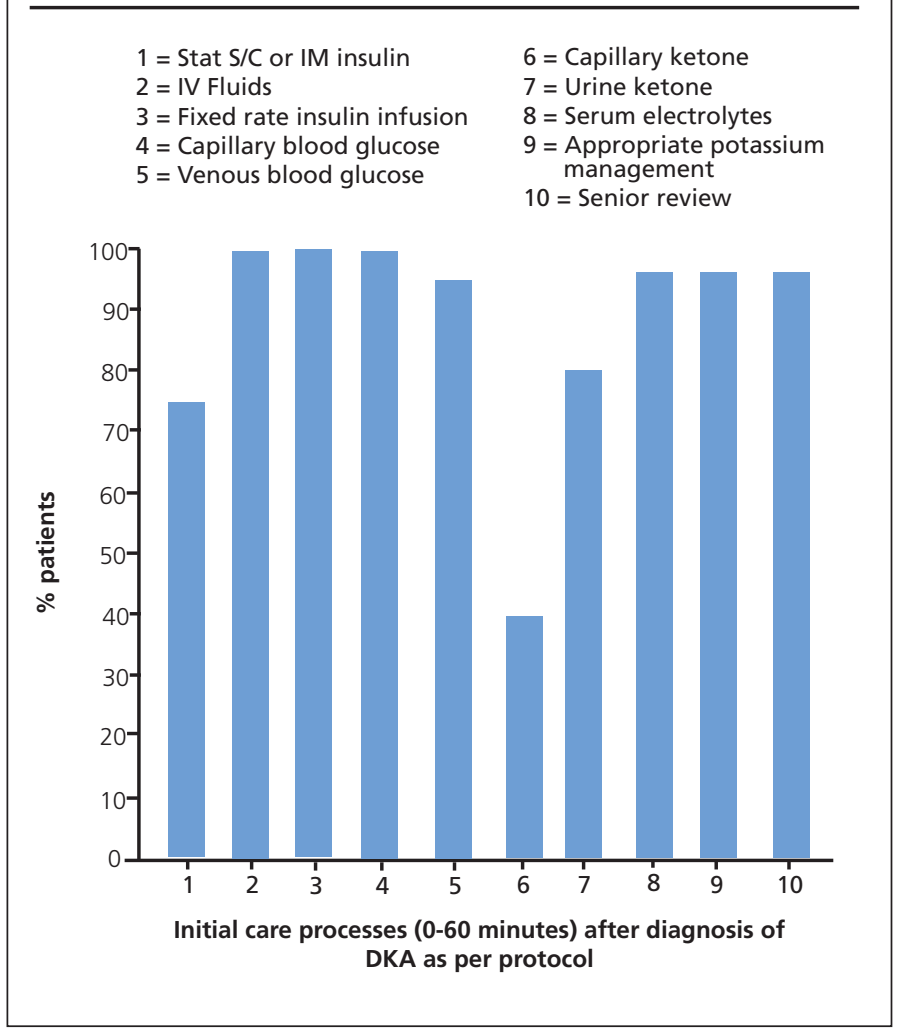

$10 \%$ dextrose prescribed when CBG dropped to $<14 \mathrm{mmol} / \mathrm{L}$ and this was consistent in the patients who experienced hypoglycaemia. Continuation of long-acting insulin analogues occurred in $30 \%$ of patients managed according to DKA protocol and was not correlated to the development of hypoglycaemia $(r=0.20, p=0.16)$.

\section{Other aspects of management}

Intravenous bicarbonate was not prescribed during any DKA episode. Intravenous phosphate (not advocated in the protocol) was administered to three patients to treat biochemical hypophosphataemia. Appropriate review of metabolic progress which included tracking of key metabolic parameters such as fluid resuscitation, intravenous insulin therapy, regular electrolyte and glucose monitoring, as per proforma occurred in $34(68 \%)$ patients. Median time to resolution of DKA was 12 hours 6 minutes. The introduction of blood ketone meters occurred in a phased manner during the audit period. When available, 26 (52\%) patients had blood ketones measured on admission. Median time to FRIII in these patients was 40 minutes compared with 52 minutes for patients in whom blood ketone levels were not checked. Fourteen patients had blood ketone levels monitored over the 24 hour period and, compared with patients who did not receive blood ketone monitoring, there were no differences in time to resolution of DKA or length of stay (refer to UHL guidelines for blood ketone measurement in the management 
Figure 2. Guidelines for blood ketone measurements in the management of DKA and in patients at risk of DKA

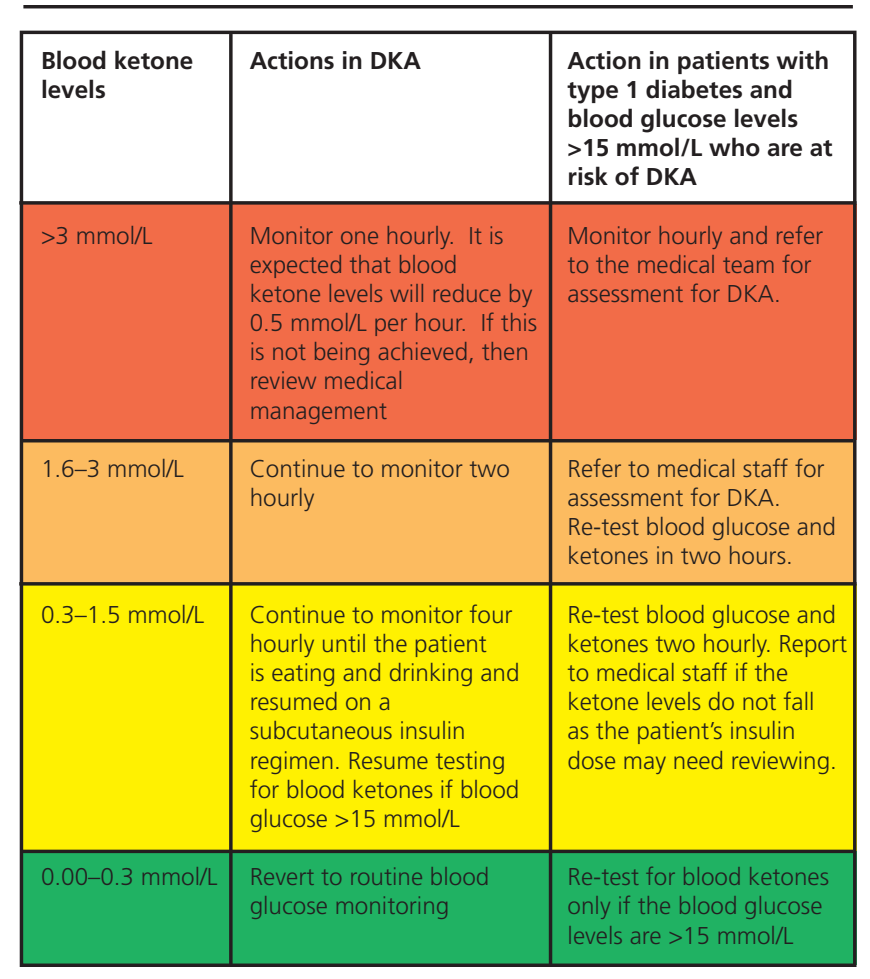

Adapted from UHL guidelines: Author Dr Kath Higgins, Diabetes Consultant; Medical CBU/Acute Division April 2012

of DKA, Figure 2). Median time to subcutaneous insulin from resolution was 11 hours and, in $66 \%$ of patients, changing from FRIII to a subcutaneous insulin regime was inappropriately managed with the most common reason being an inordinate delay in switching to a subcutaneous regimen despite resolution of DKA. Forty three (86\%) patients were reviewed by the diabetes specialist team during admission and, of the seven patients who did not receive a review, two patients self-discharged contrary to medical advice and, in the remaining five patients, no clear reasons were identified. Psychological support was not offered to any patient during hospital admission.

\section{Complications and mortality}

Forty-four percent of patients experienced either hypoglycaemia or hypokalaemia and $12 \%$ experienced both. There were no deaths directly related to DKA or due to management of complications. Three elderly patients (aged 75, 76 and 90 years) died during the index admission due to intercurrent illness diagnosed as aspiration pneumonia, pneumonia and myocardial infarction, respectively.

\section{Length of stay and follow-up}

Median length of stay was 2 days and $40 \%$ of patients had documented follow-up within 30 days from discharge. Length of stay was significantly correlated with increasing age $(r=0.28$, $p=0.04)$ and negatively correlated with frequency of metabolic review $(r=-0.29, p=0.03)$.

\section{Discussion}

This retrospective audit of management of DKA in adults since the introduction of the JBDS guidance in 2010 has demonstrated three key findings. First, good adherence to the immediate phase of treatment (0-60 minutes) with prompt diagnosis and initiation of intravenous fluids and FRIII. Secondly, a high percentage of patients received appropriate and timely senior review. Finally, among individuals in whom blood ketone levels were checked in the emergency department on admission, the median time to initiation of treatment was shorter compared with those diagnosed with urinary ketone measurements.

Of concern is the finding that $44 \%$ of patients experienced either hypoglycaemia or hypokalaemia and $12 \%$ experienced both during on-going management. Occurrence of hypokalaemia was related to poor adherence to protocol guidance on potassium supplementation and frequency of venous blood gas (and hence potassium) checks. Hypoglycaemia occurred despite 10\% dextrose being initiated appropriately in $80 \%$ of patients. It is interesting to note that the median time to hypoglycaemia occurring from diagnosis was longer than the median time to resolution of DKA, suggesting that FRIII was continued inappropriately in some patients. It is likely that this would contribute to the occurrence of hypoglycaemia, particularly in the latter stages of the management. This hypothesis is supported by the finding that the switch from FRIII to subcutaneous insulin regime was managed appropriately in only $34 \%$ of patients and median time to initiation of subcutaneous regime from resolution was 11 hours. Encouragingly, our audit demonstrated that DKA can be treated effectively with a median length of stay of 2 days (mean 3.3 days) and with no mortality associated with DKA or as a consequence of management. Length of stay was affected by increasing age and frequency of metabolic review during management.

Eighty-six percent of patients in this audit were reviewed by the diabetes specialist team prior to discharge and $40 \%$ were offered follow-up appointments within 30 days of discharge. Involvement of the diabetes specialist nurse team as standard in-patient care for adults with diabetes is vital and evidence suggests that it reduces length of stay and improves safety. ${ }^{12,13}$ Furthermore, JBDS guidance suggests that patients admitted with DKA should receive psychological support prior to discharge. However the advisory group set up to formulate the Best Practice Tariff (BPT) for DKA advised that inclusion of psychological support within the BPT was "a step too far" and that the challenges seemed insurmountable. ${ }^{14,15}$

The results of our audit are consistent with similar reports from other hospitals in the UK. Audit results from hospitals across the UK have shown that, in the first 60 minute period, initiation of intravenous fluids occurred in 39-73\%, FRIII initiation in 25-78\%, hypokalaemia occurred in 30-46\%, hypoglycaemia in 10-40\%, continuation of basal insulin in $30-58 \%$ and diabetes nurse specialist review in $57-86 \%$ of patients. ${ }^{16-21}$ A survey of the 


\section{Key messages}

- Diabetic ketoacidosis continues to be associated with significant morbidity

- The JBDS guidance recommends fixed rate intravenous insulin infusion, hourly blood ketone monitoring, continuation of basal subcutaneous insulin and regular review of fluid status and electrolyte replacement as mainstays of treatment

- Hypokalaemia and hypoglycaemia are both relatively common responses to treatment; regular review and caution are advised

management of DKA in 13 intensive care units across the east of England in 2011, showed that a majority were using local guidelines and had not yet adopted the JBDS guidelines. ${ }^{22}$ Only four units used FRIII, a minority advocated continuation of long-acting insulin analogues and both $0.9 \%$ sodium chloride solution and Hartmann's solution were equally preferred as resuscitation fluids. ${ }^{22}$ Furthermore, a study from a university hospital in south west England showed that there was a need for more understanding of DKA protocols by nursing staff, regular reassessments of patients by doctors after initial admission and management, improvements in continuity of care and accessibility of junior doctor staff in busy hospitals. ${ }^{23}$ Since the initial stages of DKA are mostly managed in acute medical and emergency units and rapid change over of staff is a recognised problem, it is imperative that ongoing training and awareness of the revised DKA guidelines is a continual process. Education for both health care providers and patients at risk for DKA may help to increase awareness and decrease the overall prevalence of this condition. ${ }^{24}$

In summary, this audit reinforces a growing evidence base that guideline adherence in the management of DKA is good in the immediate stage of treatment; however inadequate metabolic monitoring, iatrogenic hypoglycaemia and poor fluid/electrolyte management remain areas of concern. Our audit also shows that a high proportion of patients received input from the diabetes specialist nurse team with reduced length of stay and no recorded mortality due to DKA or related complications. Subsequent to our audit report, we have published a Trust wide Education Newsletter for Inpatient Diabetes (ENID, Issue 1) which focused on the management of DKA supplemented by key recommendations including the tag line "Don't forget the $K^{+}$in DKA" and "DKA is DK+ $A$ - Diabetic Keto Acidosis Insulin Saline / Senior review Dextrose $\mathrm{K}^{+}$(potassium) Assessment for metabolic progress and complications". Our own recommendations as a result of this audit include a strengthened programme of teaching and education for nursing and medical staff (including ThinkGlucose), focus on timely and appropriate potassium supplementation, telephone contact by the specialist team with the patient within 48 hours of discharge, audit of DKA management on the labour ward and plans for re-audit in two years after implementation of these recommendations.

Finally, in recognition of the need to understand the national picture with regard to management of diabetic ketoacidosis and as part of a "service improvement exercise", $A B C D$ with the backing of JBDS and Diabetes UK are currently undertaking a nationwide audit which should help to inform and aid any necessary revisions to the current national DKA guidelines. ${ }^{25}$

Contributors All authors contributed significantly to the paper, and have read and consented to its submission.

Conflict of interest No conflicts of interest declared.

Funding None.

Acknowledgements Elizabeth Hackett, Diabetes Specialist Pharmacist; Helen Atkins, Inpatient Diabetes Nurse Specialist; Dr Martin Wiese, Consultant Emergency Medicine

\section{References}

1. Liu CC, Chen KR, Chen HF et al. Trends in hospitalization for diabetic ketoacidosis in diabetic patients in Taiwan: Analysis of National Claims Data, 1997-2005. J Formosan Med Assoc 2010;109;725-34. http://dx.doi.org/10.1016/S0929-6646(10)60117-9

2. Pinkney JH, Bingley PJ, Sawtell PA, Dunger DB, Gale EAM. Presentation and progress of childhood diabetes mellitus: a prospective populationbased study. Diabetologia 1994;37:70-4. http://dx.doi.org/10.1007/BF00428780

3. Dunger DB, Sperling MA, Acerini CL et al. ESPE/LWPES consensus statement on diabetic ketoacidosis in children and adolescents. Arch Dis Child 2004;89:188-94. http://dx.doi.org/10.1136/ adc.2003.044875

4. Patterson CC, Dahlquist GG, Gyürüs E, Green A, Soltész G. Incidence trends for childhood type 1 diabetes in Europe during 1989-2003 and predicted new cases 2005-20: a multicentre prospective registration study. The Lancet 2009;373:2027-33. http://dx.doi.org/10.1016/S0140-6736(09)60568-7

5. Lokulo-Sodipe K, Moon RJ, Edge JA, Davies JH. Identifying targets to reduce the incidence of diabetic ketoacidosis at diagnosis of type 1 diabetes in the UK. Arch Dis Child 2014;99:438-42. http://dx.doi.org/10.1136/archdischild-2013-304818

6. The Health and Social Care Information Centre. National Diabetes Audit Paediatric Report 2009-2010.Available at: http://www.ic.nhs.uk/webfiles/Services/NCASP/Diabetes/2009 annual report documents/ NHSIC_National_Diabetes_Paediatric_Audit_Report. pdf . Last accessed 2nd June 2014

7. Joint British Diabetes Societies Inpatient Care Group. The Management of Diabetic Ketoacidosis in Adults 2010. http://www. diabetologistsabcd.org.uk .

8. Dabelea D, Rewers A, Stafford JM et al. Trends in the prevalence of ketoacidosis at diabetes diagnosis: The SEARCH for Diabetes in Youth Study. Pediatrics 2014;13:e938-e945. http://dx.doi.org/10.1542/peds.2013-2795

9. Health and Social Care Information Centre. National Diabetes Inpatient Audit 2012. http://www.diabetes.org.uk

10. Joint British Diabetes Societies Inpatient Care Group. The Management of Diabetic Ketoacidosis in Adults. Revised September 2013. http://www. diabetologists-abcd.org.uk/subsite/JBDS

11. UHL Inpatient Diabetes Steering Group. Guidelines for the Management of Diabetic Ketoacidosis (DKA) in Adults. http://www.leicestershirediabetes.org.uk . 2012.

12. Davies M, Dixon S, Currie CJ, Davis RE, Peters JR. Evaluation of a hospital diabetes specialist nursing service: a randomized controlled trial. Diabet Med 2001;18:301-07. http://dx.doi.org/10.1046/j.1464-5491.2001.00470.x

13. Flanagan D, Moore E, Baker S, Wright D, Lynch P. Diabetes care in hospital-the impact of a dedicated inpatient care team. Diabet Med 2008, 25:147-51. http://dx.doi.org/10.1111/j.1464-5491.2007.02326.x

14. Price H, Thomsett K, Newton I, Alderson S, Hillson R. Developing best practice tariffs for diabetic ketoacidosis and hypoglycaemia. Practical 
Diabetes 2013;30:6-8. http://dx.doi.org/10.1002/pdi.1731

15. Department of Health. Payment by Results (PbR) in the NHS. Available at: https://www.gov.uk/government/collections/payment-by-results2013-14

16. Chakera AJ, Brooks AM, Jones AG, Foster JAH, Carpenter AB. Can we use new national guidelines regarding the management of diabetic ketoacidosis to improve care in a teaching hospital? Diabet Med 2011;P386;28(Suppl.1),32-203.

17. Bondugulapati LNR, Phyu CHO, Irrinki L, Dixon A, Griffiths C. Audit on the management of diabetic ketoacidosis in a district general hospital. Diabet Med 2013;P166;30(Suppl.1),30-201.

18. Manikandan RM , Abel J, Verdaguer R, Rajendran R, Masding MG. Management of diabetic ketoacidosis improves with implementation of care pathway. Diabet Med 2012;P307;29(Suppl.1),30-177.

19. Parthasarathy S, Ellis K, Smith A, Crown A, Vaughan N. Management of diabetic ketoacidosis: comparison against standards using an online audit tool. Diabet Med 2014;P160;31(Suppl.1),28-183.

20. Kurdi H, Pinto LP, Smeeton FJ. An audit of the management of diabetic ketoacidosis in a district general hospital after introduction of the Joint British Diabetes Societies guidelines. Diabet Med 2014;P359;31 (Suppl.1),28-183.
21. van Zeller C, Milner T, Herring R, Russell-Jones D. A paradigm shift in the management of diabetic ketoacidosis: the introduction of the Joint British Diabetes Societies 2010 guideline into the Royal Surrey County Hospital. Diabet Med 2014;P450;31(Suppl.1),28-183.

22. Rudd B, Patel K, Levy N, Dhatariya K. A survey of the implementation of the NHS diabetes guidelines for management of diabetic ketoacidosis in the intensive care units of the East of England. J Intensive Care Soc 2013;14:61-4.

23. Thomson-Moore A, Low A, Williams J, Bradley K, Thorogood N. Impact of the 2010 Diabetic Ketoacidosis (DKA) Guidelines (based on the 2010 Joint British Diabetes Societies Inpatient Care Group Standards of Care) and IV Insulin and Fluid Prescription Chart on DKA management at University Hospitals Bristol NHS Foundation Trust. Diabet Med 2012; P318;29(Supp.1),30-177

24. Brown CW, Watson WA, Morrison IG, MacLennan KY. Novel methods of teaching diabetic ketoacidosis (DKA) diagnosis and management in a clinical curriculum: Sim-DKA. Practical Diabetes 2014;31:207-11. http://dx.doi.org/10.1002/pdi.1868

25. $A B C D$ Administration. Please join the national audit of DKA management 2014; http://www. diabetologists-abcd.org.uk.

\section{Casting Course for the Diabetic Foot 2015}

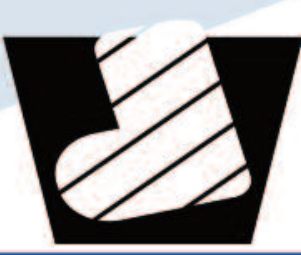

\section{About the course}

It is aimed at health professionals who are working as part of an MDFT who wish to learn how to cast in order to treat neuropathic foot complications more effectively.

- The course aims to standardise care and improve clinical outcomes for patients presenting with a neuropathic diabetic foot complications

- Standardised techniques and terminology used in casting to ensure replicable results

- Step by step guidance to manufacturing a cast

- The course is both theory and practical sessions on the manufacture of removable and a non-removable below knee cast and cast boot

- Ongoing support can be provided by the course facilitators to help get you started

Rachel Berrington and Catherine Gooday Diabetic foot leads from University Hospitals of Leicester and Norfolk \& Norwich University Hospital are organising this program on behalf of a multidisciplinary casting expert group.
How to cast a diabetic foot to achieve optimal outcomes for our patients! 